\title{
Research Paper \\ Examining the Effectiveness of Hope-Based Group Training on the Life Quality of the Elderly People
}

\author{
Meisam Ghazi Mohseni ${ }^{1},{ }^{*}$ Ali Akbar Soleimanian ${ }^{1}$, Ahmad Heidarnia $^{1}$
}

1. Department of Counseling, Faculty of Humanities, University of Bojnord, Bojnord, Iran

Cltation: Ghazi Mohseni M, Soleimanian AA, Heidarnia A. [Examining the effectiveness of hope-based group training on the life quality of the elderly People (Persian)]. Iranian Journal of Ageing. 2016; 11(2):300-309. http://dx.crossref.org/10.21859/sija-1102300

of: http://dx.crossref.org/10.21859/sija-1102300

Received: 28 Apr. 2016 Accepted: 21 Jun. 2016

Key words: Hope-based training, Quality of life, Elderly

\begin{abstract}
Objectives The rapid growth of the population, especially in developing countries, has led to adulthood phenomenon, which poses as one of the challenges of the public health. Therefore, it is necessary to pay close attention to this age group. This age group mostly suffers from psychological and physical problems. Psychologists have devised a variety of measures for tackling with these problems including disappointment, depression, and loneliness. However, after the emergence of positive psychology, scholars, instead of focusing on negative experience, deal with positive constructs such as self-control, spirituality, happiness, and hope. Therefore, this study attempts to investigate the effect of hope-based group training on the quality of life of the elderly.

Methods \& Materials The present study adopted a semi-experimental design with pretest-posttest and group control. The study population comprised of all the elderly people in "Omid-e-Golestan Daily Center" in Minoodasht. Thirty participants were randomly selected out of 60 individuals, and divided into two groups namely control group and experimental group, each containing 15 participants. The 26-question Short Form Quality of Life Inventory developed by WHO, was used to collect the required data. After implementing the pretest, the experimental group received hope-based group training-for 1.5 hours in 8 sessions, once a week. The pretest was then repeated for the two groups, and the data was analyzed using the covariance analysis.

Results The findings of the study showed that hope-based group training not only enhanced the quality of life but also improved the physical dimension of the elderly in the experimental group .Furthermore, a significant improvement was noted in the psychological, environmental, and social life quality of the elderly $(P \leq 0.05)$.

Conclusion Based on the results of the study, it was concluded that hope-based group training could significantly enhance the quality of life of the elderly. With the rapid increase in the number of elderly, hope-based group training is highly recommended.
\end{abstract}




\title{
بررسى اثربخشى آموزش تروهى اميدمحور بر ارتقاى كيفيت زندكى سالمندان
}

\author{
ميثم قاضىمحسنى'، "علىاكبر سليمانيان'، احمد حيدرنيا'

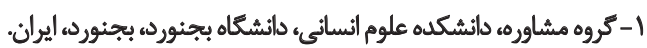

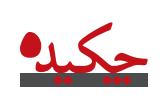

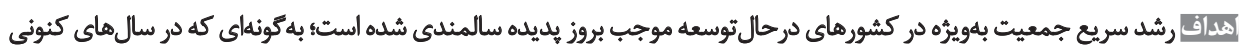

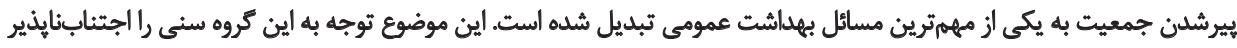

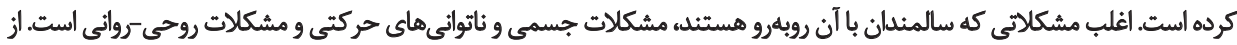

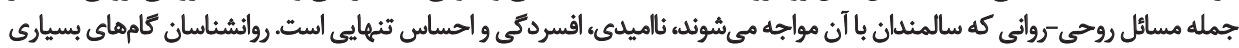

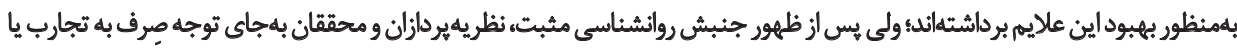

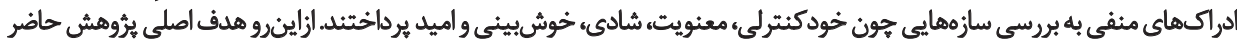

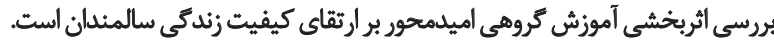

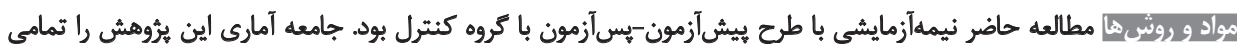

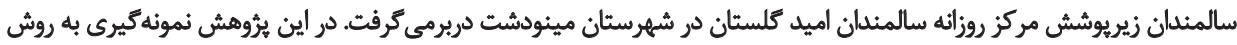

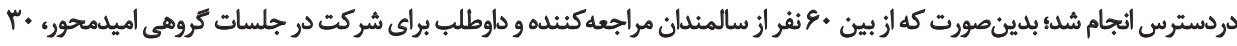

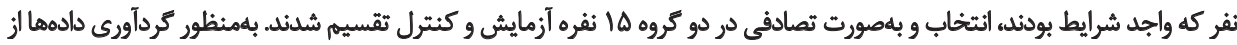

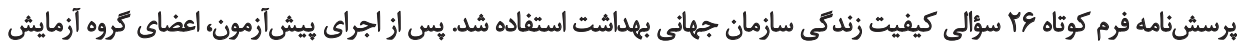

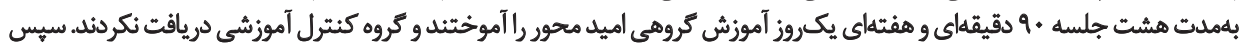

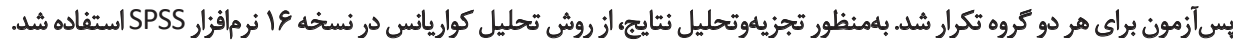

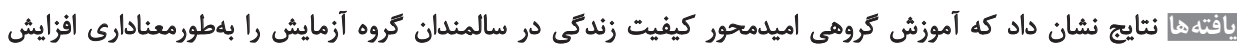

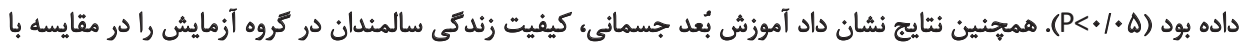

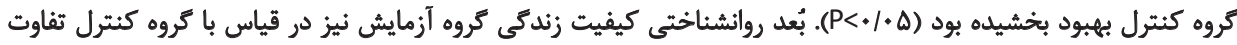

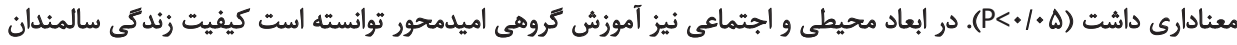

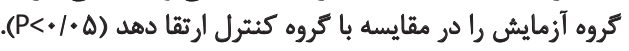

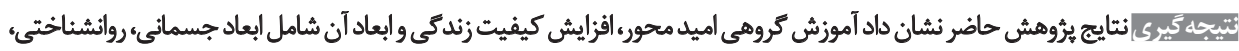

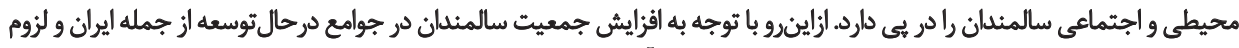

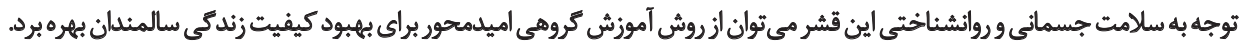

تاريخ دريافت: 9 ارديبهشت هوبها

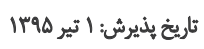

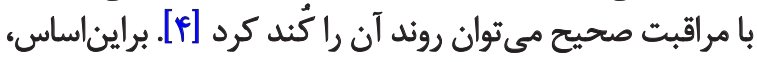

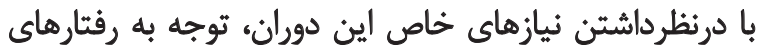

سالمندى دوران حساسى از زندگى است و توجه به مسائل

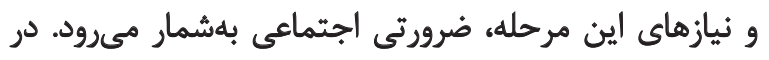

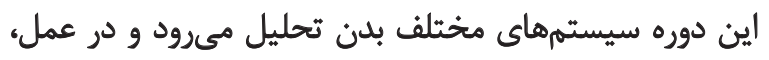

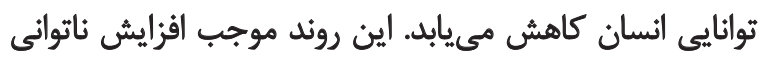

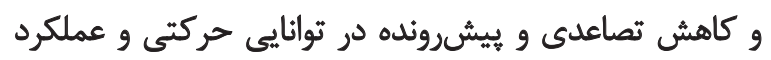


بلنظر ميرسد اميد عامل ههمي در اثربخشى اقدامات

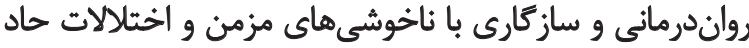

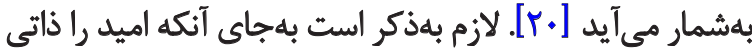

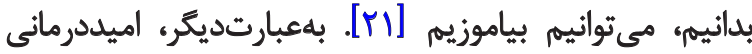

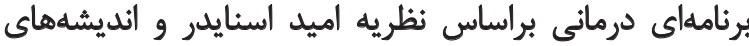

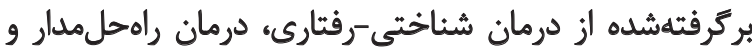

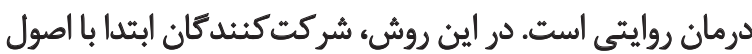

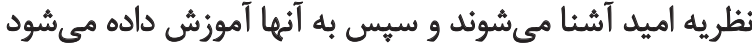

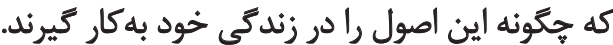

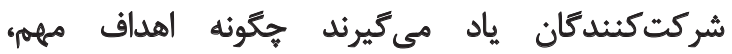

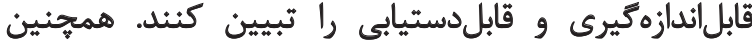

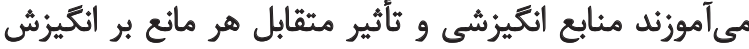

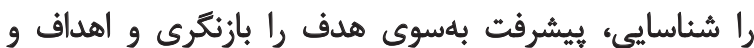

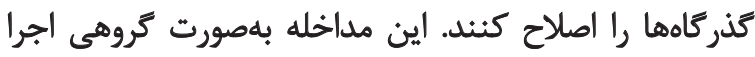

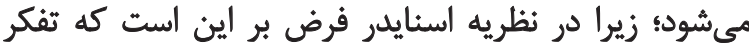

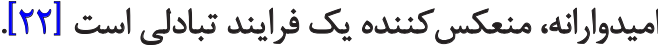
علاوهبراين، مشاوره و آموزش كروهي مى تواند باند باند سلامت

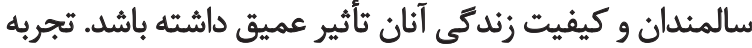

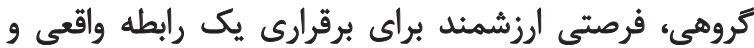

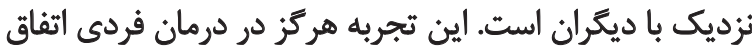

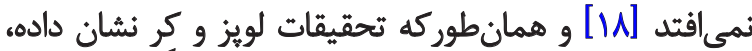

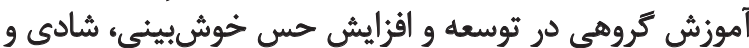

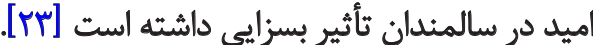

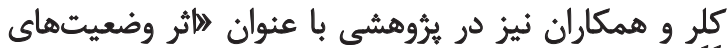

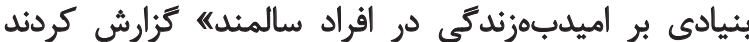

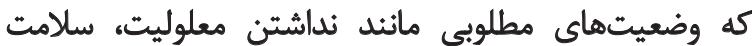

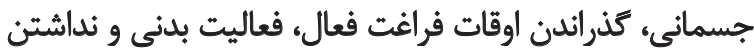

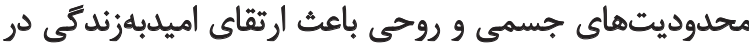

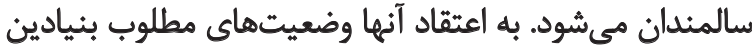

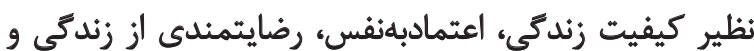

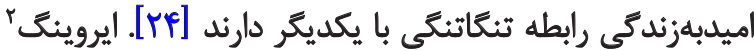

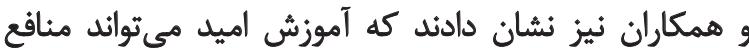

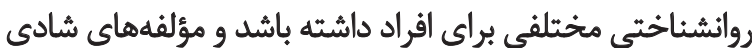

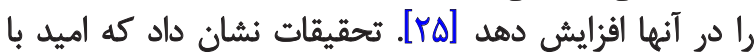

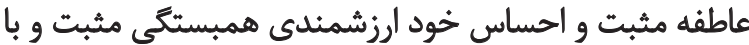

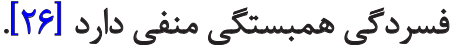

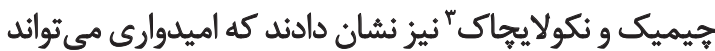

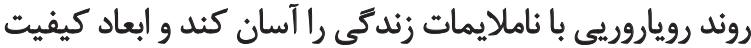

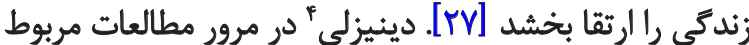

2. Irving

3. Chimick and Nekolaichak

4. Denizli

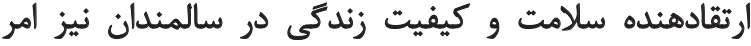

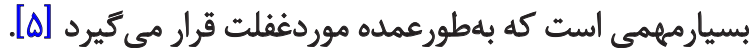
كيفيت زندكى، شاخصى اساسى محسوب مي شود و اززآنجاكه

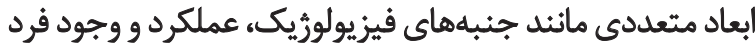

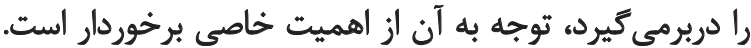

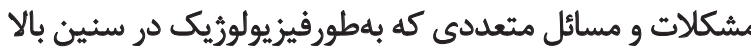

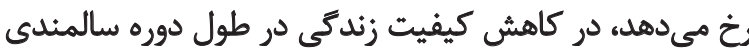

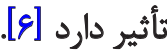

سازمان بهداشت جهاني اعلام كرده است كه در همه كشورها

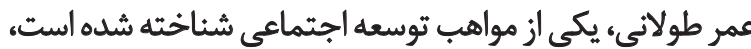

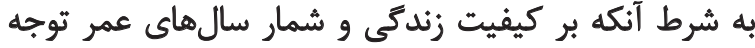

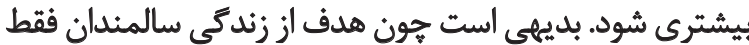

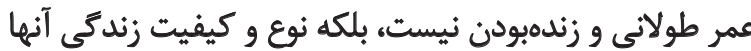

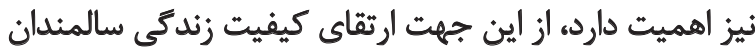

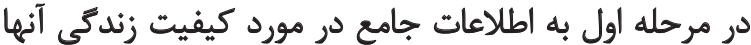

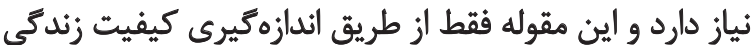

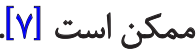

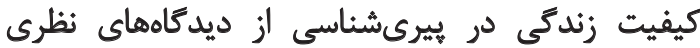

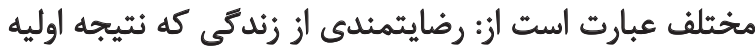

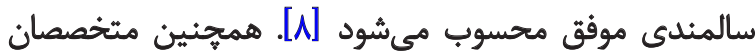

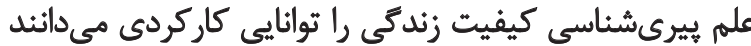

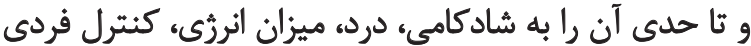

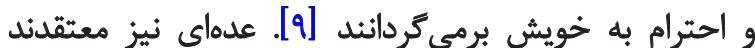

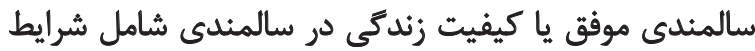

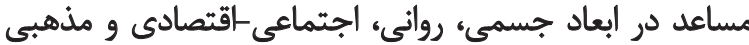

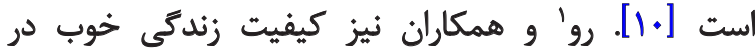

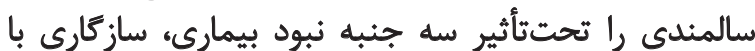

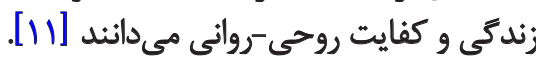

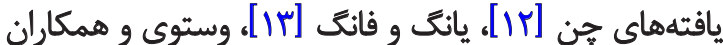

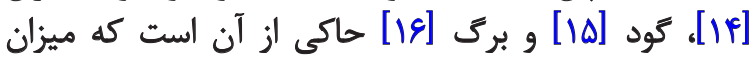

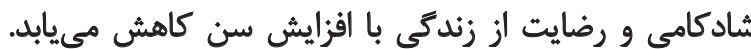

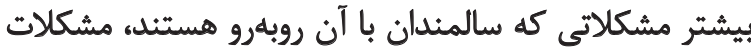

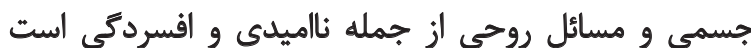

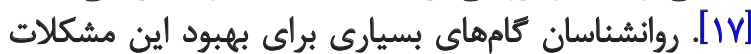

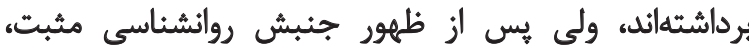

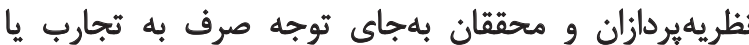

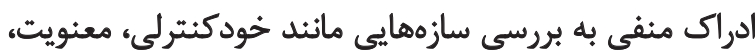

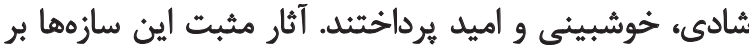

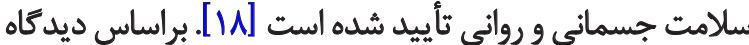

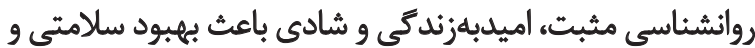

رفع تنش مى مئود [19]. 
كزارش كرده است. همجنين نتايج هربوط به روايى همزمان

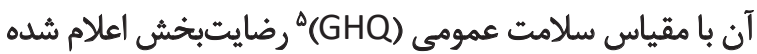

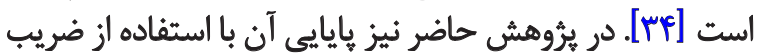

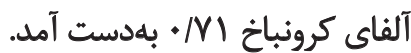

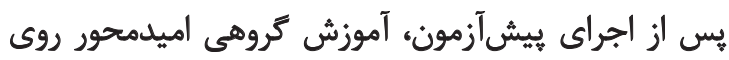

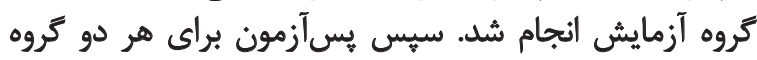

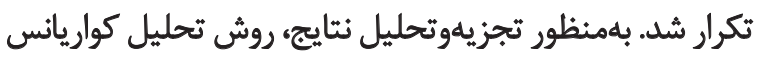

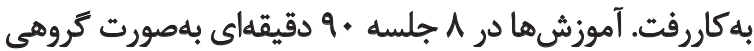

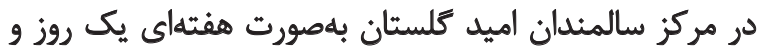
براساس يثروهش هاى اسنايدر طراحى و اجران شاند.

• در جلسه اول يرسشنامه كيفيت زندكى اجرا و سيس ساختار

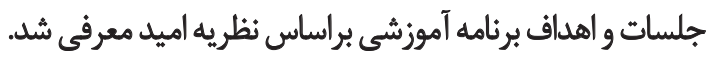

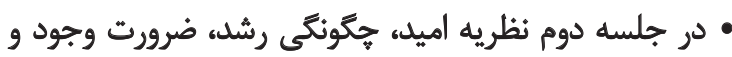
تأثير آن بر كيفيت زند دومى انجام شد.

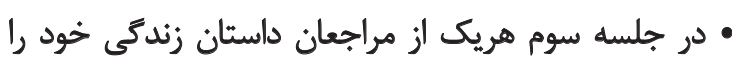

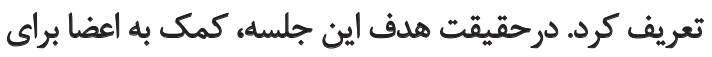

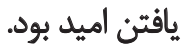

• جلسه جهارم تبيين داستان با براساس سه مؤلفه اصلى نظريه

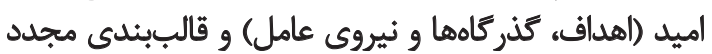

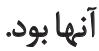

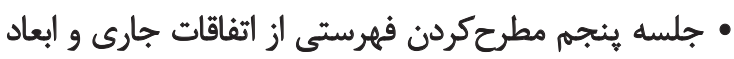

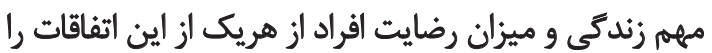
دربر مانشت.

• در جلسه ششم افراد به انتخاب اهداف مناسب و مطرحكردن

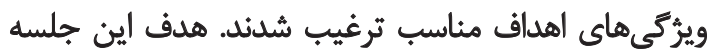

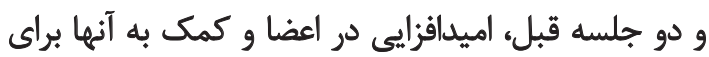
افزايش اميده يس إز يافتن آن بود.

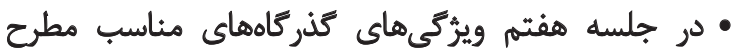

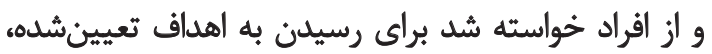
راهكار هاى مئاسب انتخاب كنئد.

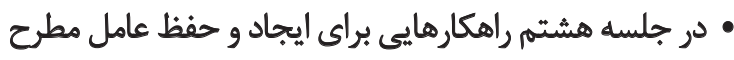

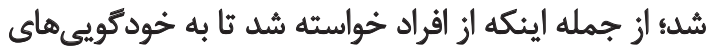

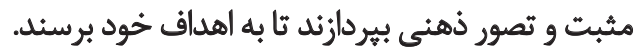

Latợ

بلمنظور تجزيهوتحليل يافتههاى ثرُوهش حاضر، ابتدا

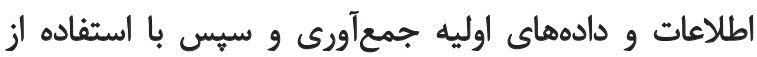

5. General Health Questionnaire (GHQ)
به اميد معتقد است سطوح بالاى اميد با سلامت جسمى و ورانو

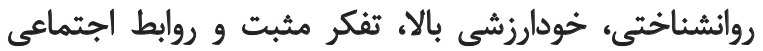

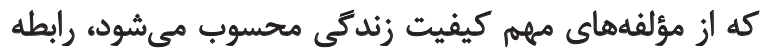

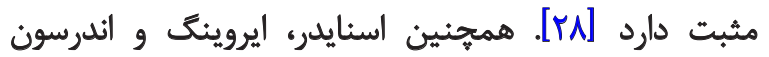

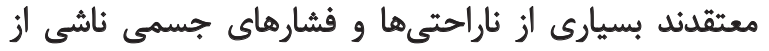

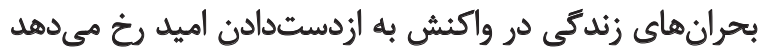

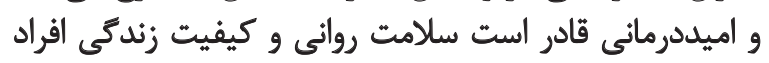

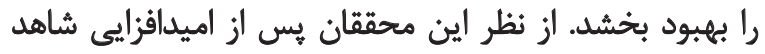

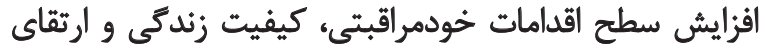
سلامت عمومى در افراد خواهيم بود [باتئ.

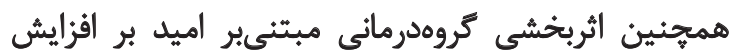

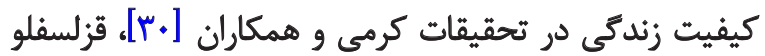

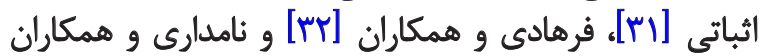

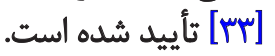

بنابراين با توجه به نتايج مطالعات مذكور، افزايش جمعيت ائل دايت

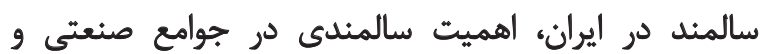

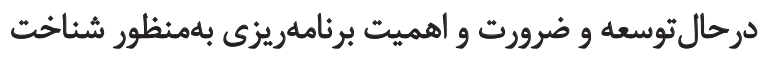

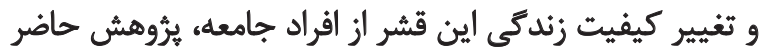

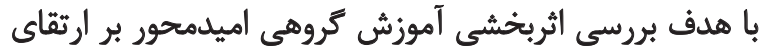

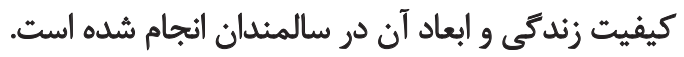

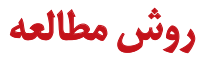

مطالعه حاضر از لحاظ هدف، كاربردى و از نظر نحوه

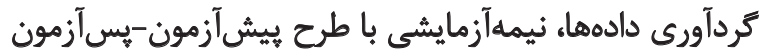

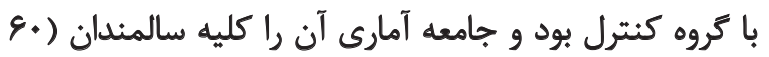

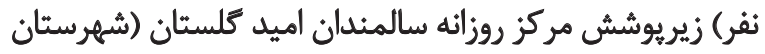

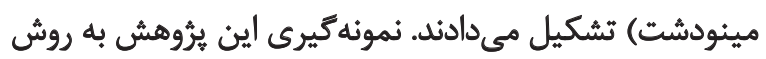

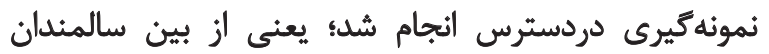

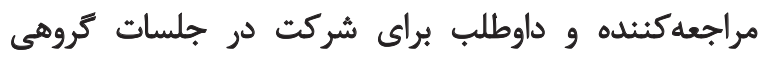

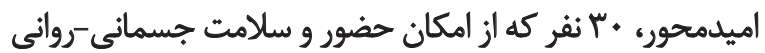

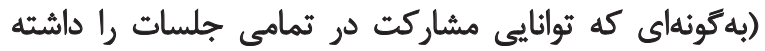

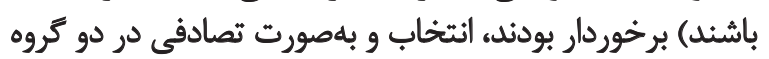
آزمايش و كنترل جايكزين شدند. باند.

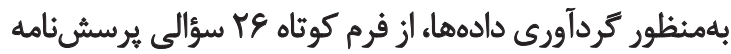

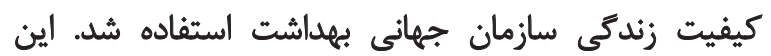

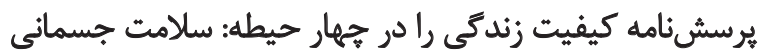

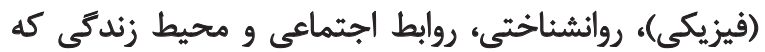

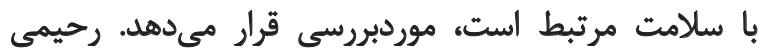
(Y..V)

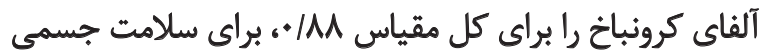

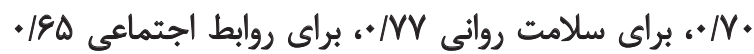

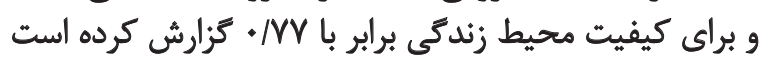

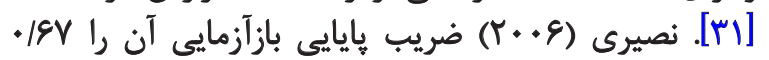


جدول ا. ميائكين سنى و توزيع سطح تحصيلات افراد كروه آزمايش و كنترل.

\begin{tabular}{|c|c|c|c|c|c|}
\hline درصد & فراوانى & وضعيت تحصيلات & أتحرافمعيار & ميانكين سنى & So \\
\hline NEISE & ir & ل بى سواد & \multirow{3}{*}{$\Delta / \varepsilon$} & \multirow{3}{*}{$s q / 4}$. & \multirow{3}{*}{ كروه أزمايش } \\
\hline $1 \pi / \pi$ & r & ابتدايع & & & \\
\hline - & - & راهنمايي & & & \\
\hline$V N / M r$ & 11 & ل & \multirow{3}{*}{ NF. } & \multirow{3}{*}{$E q / A$. } & \multirow{3}{*}{ كروه كثترل } \\
\hline sles & 1 & ابثدايي & & & \\
\hline r. & r & راهنمايي & & & \\
\hline \multicolumn{2}{|c|}{ كـ المن } & \multicolumn{4}{|c|}{ قدول r. ميانكين و ائحراف استاندارد نمره كيفيت زندكى و ابعاد آن در كروههاى آزمايش و كنترل. } \\
\hline \multicolumn{2}{|c|}{ 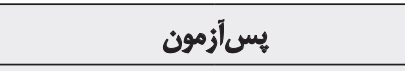 } & \multicolumn{2}{|c|}{ بيش آزمون } & \multirow{2}{*}{$\log _{5}$} & \multirow{2}{*}{ ابعاد } \\
\hline انحرافهعيار & مياكين & انحرافـععيار & مياتكين & & \\
\hline$p / \Delta q$ & MTls. & $r / \Lambda$. & Wr. & آزمايش & \multirow{2}{*}{ جسمائى } \\
\hline$p / v q$ & $r \cdot 18$ & p/gr & $r \cdot 1 A$. & كتّرل & \\
\hline$m / T^{\prime} \mid$ & Tr/YE & $r / \cdot V$ & 11 & آزمايش & \multirow{2}{*}{ روأششناختى } \\
\hline r/ar & Wr. & $M / 1 Q$ & Wr. & كتترل & \\
\hline Veft & ir & r/ar & 1.11 & أزمايش & \multirow{2}{*}{ الجتماعى } \\
\hline$r / m$ & $9 / 9 \pi$ & $\mathrm{I} / \mathrm{W}$ & 11 & كتترل & \\
\hline r/ar & rq/p. & r/19 & $\mu \varphi / \varphi$ & آزمايش & \multirow{2}{*}{ محيطى } \\
\hline$r / 4 A$ & rf/qu & T/KE & $\mathrm{ra} / \mathrm{\Delta r}$ & كتترل & \\
\hline$N+\varepsilon$ & AV/TE & $V / \& A$ & $n / r^{*}$ & أزمايش & \multirow{2}{*}{ كيفيت زندكى كل } \\
\hline$q / 1{ }^{2}$ & $n / n$ & NI. & $V \Delta / M^{\infty}$ & كتّرل & \\
\hline
\end{tabular}

地

جدول ". نتّايج تحليل كواريائس مقايسه نمره كل كيفيت زندكى در كروهماى آزمايش و كثترل.

\begin{tabular}{|c|c|c|c|c|c|c|c|}
\hline توان أزمون & ضريب اتا & مقدار احتمال & $\mathbf{F}$ & ميانئين مجذورات & ورجه أزادى & مجموع مجذورات & \\
\hline \multirow{4}{*}{1} & \multirow{4}{*}{$\cdot M r$} & $\%$ & $V / M I$ & laiffe. & 1 & $101 f / f$. & ييش آزمون \\
\hline & & .1 .0 & $1 . T / K A$ & rift/P & 1 & MIFT/IF & ملاخله كروه \\
\hline & & - & - & r./AY & rV & $\Delta F \Delta / F$ & خطا \\
\hline & & - & - & - & r. & $1.81 \Delta H^{\prime}$ & كل \\
\hline
\end{tabular}

次

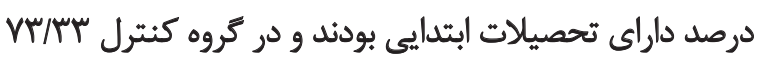

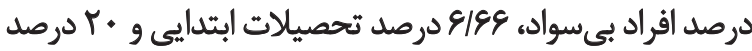

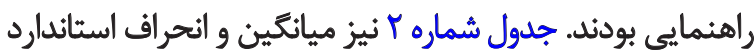

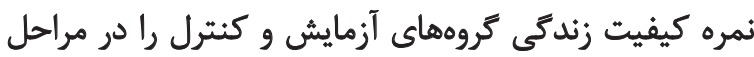

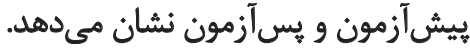
همجنان كه دادهاي جدول شماره r نشان مي دهد ميانكين

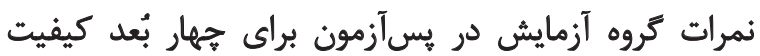

روش هاى آمارى تحليل كواريانس و تى مستقل، به آزمون

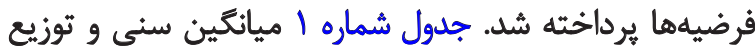

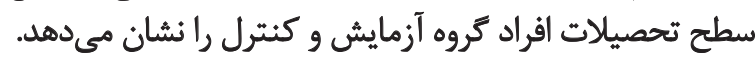
بر اساس جدول شماره 1 ميانكين سنى كروه آزمايش

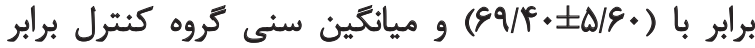

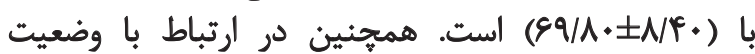

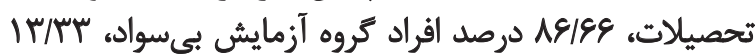


جدول †. نتايج تحليل كواريانس مثايسه ابعاد كيفيت زندگى در دو كروه كنترل و آزمايش.

\begin{tabular}{|c|c|c|c|c|c|c|c|c|}
\hline توان آزمون & ضريب اتا & مقدار احتمال & $\mathbf{F}$ & ميانكين مجذورات & درجه آزادى & مجموع مجذورات & منابع & هثغير \\
\hline \multirow{2}{*}{.$/ 9 \Delta$} & \multirow{2}{*}{.$/ M \varphi$} & $+\ldots+1$ & $r g / . r$ & $r \cdot r / A \mid$ & 1 & $r+r / \lambda I$ & ييش آزمون & \multirow{2}{*}{ جسمائى } \\
\hline & & $.1 . .1$ & $\mid E / T \Delta$ & $\mid 8 v / .$. & 1 & $\mid 8 \mathrm{~V} / .$. & مداخله كروه & \\
\hline \multirow{2}{*}{.$/ 99$} & \multirow{2}{*}{.$/ \mu T$} & $.1 . .1$ & $\mid A / \cdot$. & $1.1 / \pi 1$ & 1 & $1.1 / \pi 1$ & ييش:آزمون & \multirow{2}{*}{ روانشناختى } \\
\hline & & $\% \ldots 1$ & $r \cdot / \Delta r$ & ITNV & 1 & ITNVE & مداخله كروه & \\
\hline \multirow{2}{*}{1} & \multirow{2}{*}{$\cdot / \mathrm{AA}$} & $.1 . .1$ & $15 / 199$ & ENA. & 1 & ENA. & بيشآزمون & \multirow{2}{*}{ محيطى } \\
\hline & & $+\cdots \cdot 1$ & YNTI & $149 / T V$ & 1 & IAq/T & مداخله كروه & \\
\hline
\end{tabular}

il

جدول هـ نتايج تي مستقل در بُعد اجتماعى در دو مروه كثترل و آزمايش.

\begin{tabular}{|c|c|c|c|}
\hline مقدار احتمال & $T$ & ورجه آزادى & مثغير \\
\hline $.1 . r$ & T/RA & YM & اجتماعى \\
\hline
\end{tabular}

L

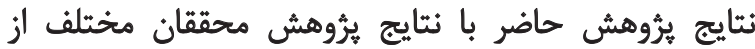

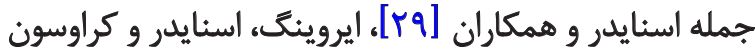

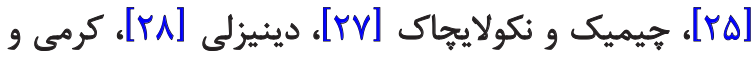

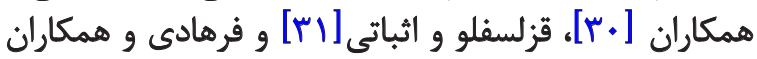

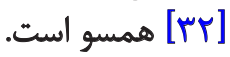

شايد بزركترين موفقيت يك شخص در زندكى برخوردارى

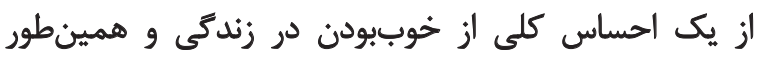

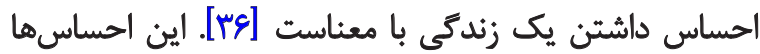

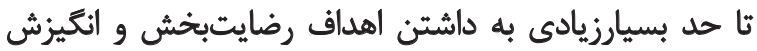

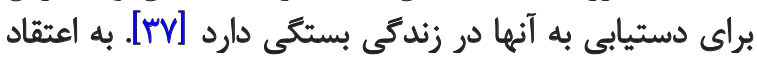

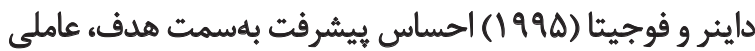

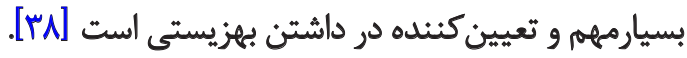

آموزش كروهي اميدمحور نيز توانسته است با ايجاد هدف و و

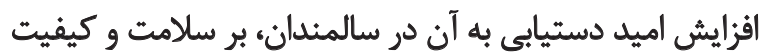

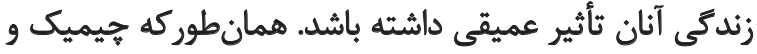

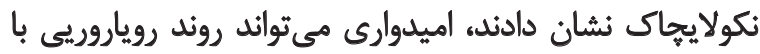

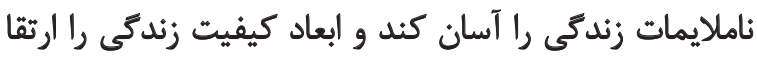

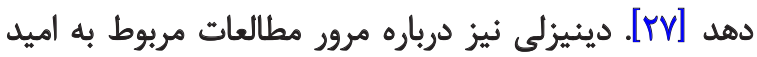

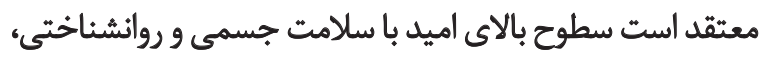

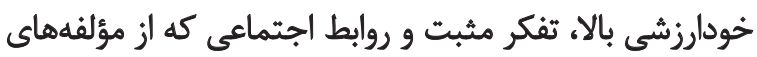

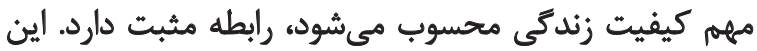

يافتهها با نتايج ئروهش كنونى همخوانى دارد [YN]

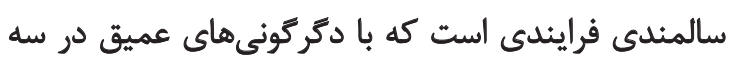

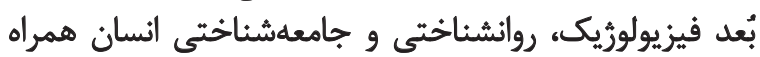

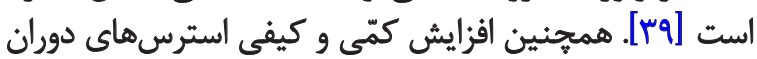

زندكى و كيفيت زندكى كل افزايش يافته است، اما نمرات كروه كنترل تغيير محسوسى نداشته و حتى كاهش دئ داشته است

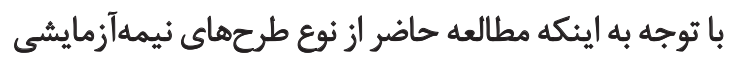

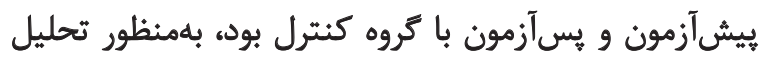

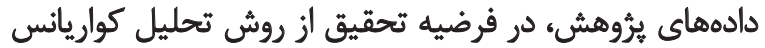

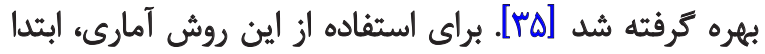

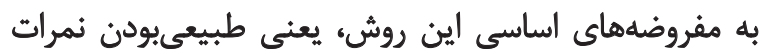

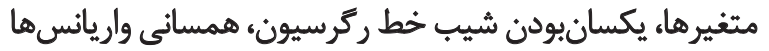

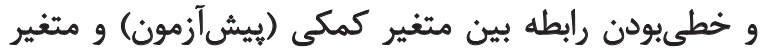
وابسته (يسآزمون)، يرداخته شد. ئرئ.

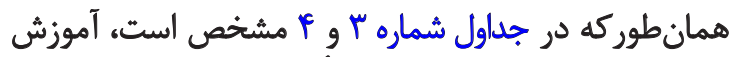

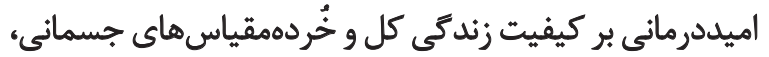

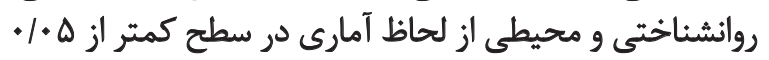

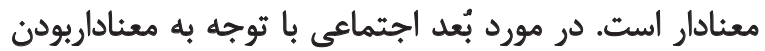

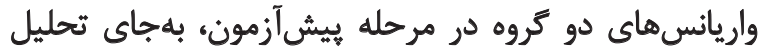

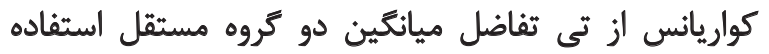

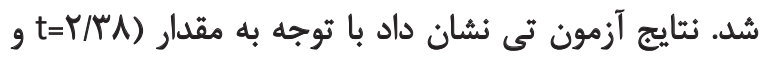

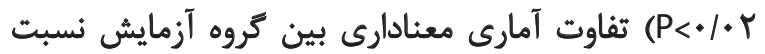

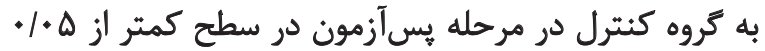
وجود دارد (جدول شماره هاه).

بحث

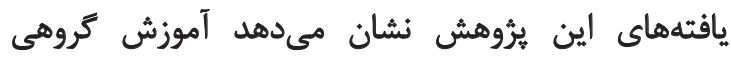

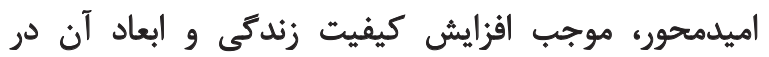

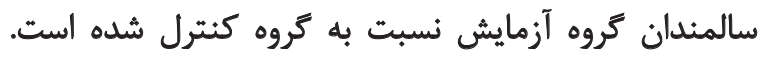


را حفظ كنند و ضمن يذيرش محدوديتهاى محيطي،

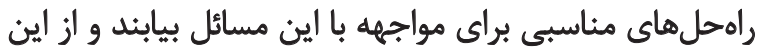
طريق همواره يويا باشند و سبك حلى حلمسئله فعالانهاى انتخاب

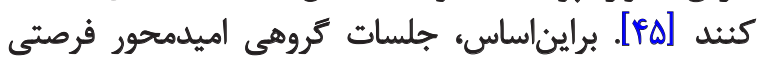

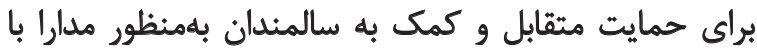

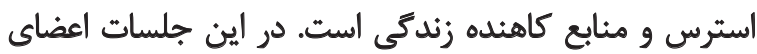

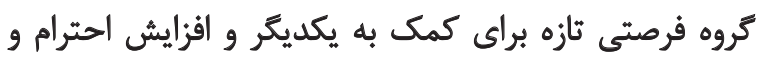

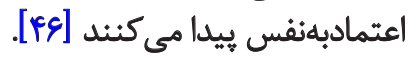

\section{نتيجيَّيرى نهايى نيق}

درمجموع ميتوان جنين نتيجهيرى كرد كه مداخلات

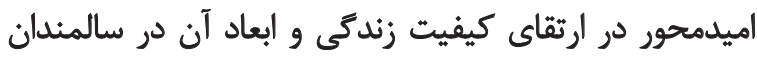

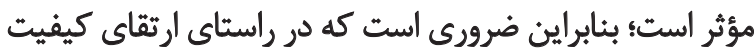

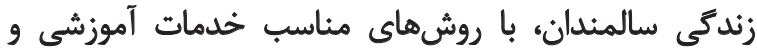
برنامههاى ارتقابخش آموزش داده شود.

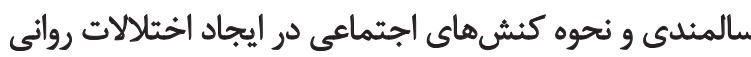

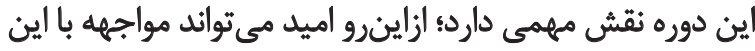

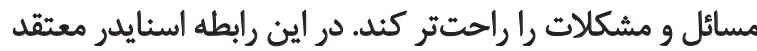

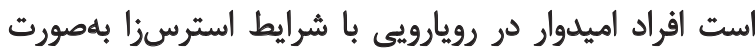

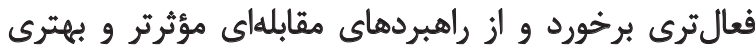

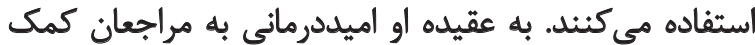

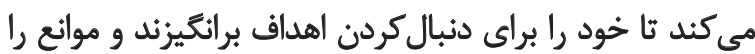

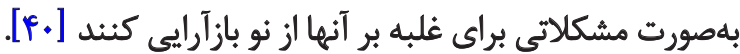

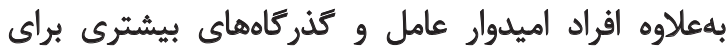

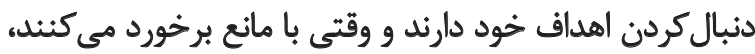

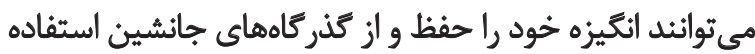

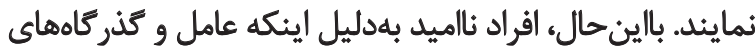

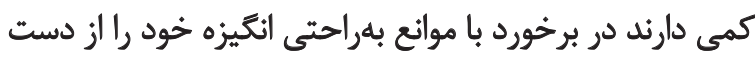

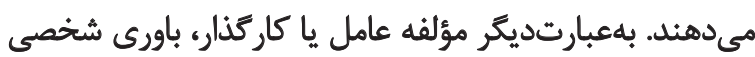

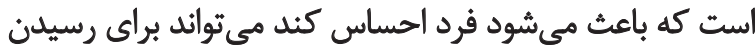

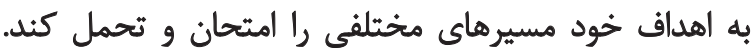

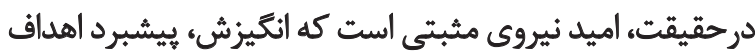

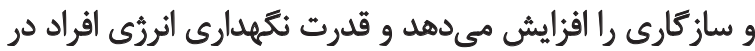

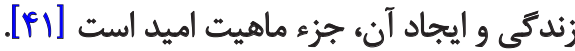

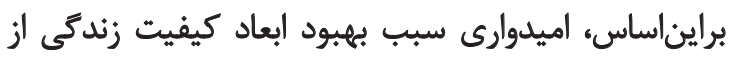

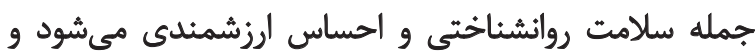

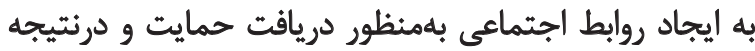

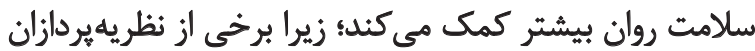

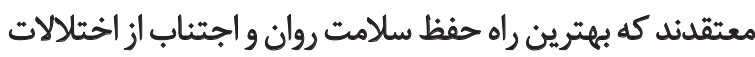

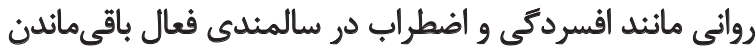

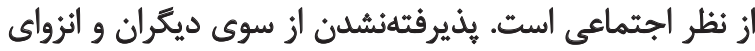

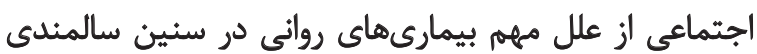

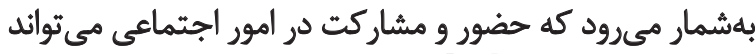

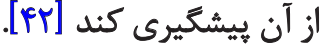

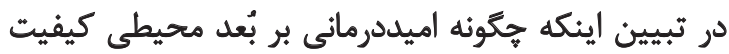

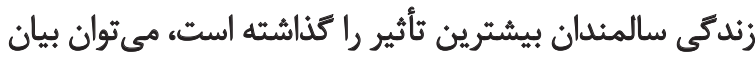

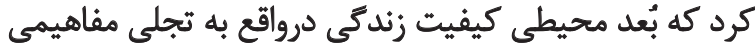

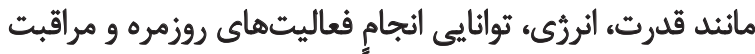

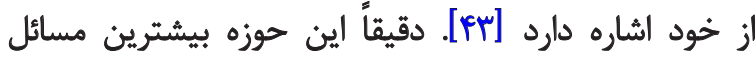

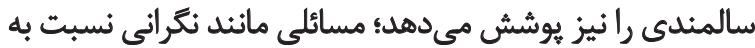

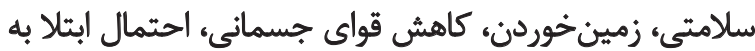

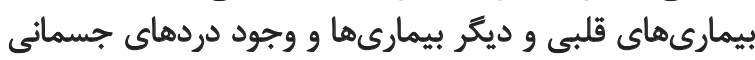

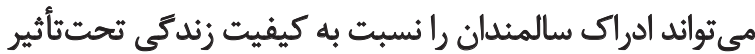

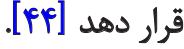

در فرايند اميددرمانى افراد تحثتأثير افزايش تفكر عامل

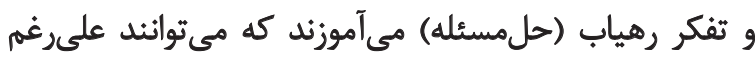

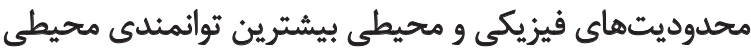




\section{References}

[1] Adib-Hajbaghery M, Aghahoseini S. The evaluation of disability and its related factors among the elderly population in Kashan, Iran. BMC Public Health. 2007; 7:261. doi: 10.1186/1471-2458-7261

[2] Glaesmer H, Riedel-Heller S, Braehler E, Spangenberg L, Luppa $M$. Age and gender specific prevalence and risk factors for depressive symptoms in the elderly: a population-based study. International Psychogeriatrics. 2011; 23(08):1294-300.

[3] World Health Organization. What are the main risk factors for disability in old age and how can disability be prevented? Geneva: World Health Organization Press; 2003.

[4] Masoudi R, Soleimani M, Hashemi NA, Qorbani M, Pour Dehkordi A, Bahrami N. [The effect of family centered empowerment model on the quality of life in elderly people (Persian)]. Journal of Qazvin University of Medical Sciences. 2010; 14(1):57-64.

[5] Simonetti JP, Ferreira JC. Coping strategies developed by caregivers of elderly with chronic diseases. Revista da Escola de Enfermagem da USP. 2008; 42(1):19-25.

[6] Young HM, Cochrane BB. Healthy aging for older women. Nursing clinics of North America. 2004; 39(1):131-43.

[7] Sajadi H, Biglarian A. [Woman aging life quality kahrizak chairty hospice (Persian)]. Payesh. 2005; 5(2):105-08.

[8] Argyle M. The psychology of happiness. Routledge: Taylor \& Francis Publication; 2013.

[9] Frisch MB. Quality of life therapy: applying a life satisfaction approach to positive psychology and cognitive therapy. New Jersey: John Wiley \& Sons Publication; 2005.

[10] Akamatsu K, Saito A, Wada T, Ishine M, Roriz-Cruz M, Okumiya K, et al. Analysis of comprehensive geriatric assessment of elderly residents in a social welfare home for the aged compared with those in a residential care home in an urban area in Japan Geriatrics \& Gerontology International. 2005; 5(1):53-58

[11] Hamidizadeh S, Ahmadi F, Aslani Y, Etemadifar S, Salehi K, Kordeyazdi R. [Study effect of a group-based exercise program on the quality of life in older men and women in 2006-2007 (Persian)]. Journal of Shahid Sadoughi University of Medical Sciences. 2008; 16(1):167-75

[12] Chen C. Aging and life satisfaction. Social Indicators Research. 2001; 54(1):57-79.

[13] Yeung GT, Fung HH. Social support and life satisfaction among Hong Kong Chinese older adults: family first? European Journal of Ageing. 2007; 4(4):219-27.

[14] Westaway MS, Olorunju SA, Rai LCJ. Which personal quality of life domains affect the happiness of older South Africans? Quality of Life Research. 2007; 16(8):1425-438.

[15] Good GA. Life satisfaction and quality of life of older New Zealanders with and without impaired vision: a descriptive, comparative study. European Journal of Ageing. 2008; 5(3):223-31.

[16] Berg AI. Life satisfaction in late life: Markers and predictors of level and change among $80+$ year olds [ $\mathrm{PhD}$ thesis]. Gothenburg: University of Gothenburg; 2008.

[17] Bailey TC, Snyder C. Satisfaction with life and hope: a look at age and marital status. Psychological Record. 2007; 57(2):233-42.
[18] Navvabinejad S. [Group counseling theories (Persian)]. Tehran: Samt; 2004

[19] You S, Furlong MJ, Felix E, Sharkey JD, Tanigawa D, Green JG Relations among school connectedness, hope, life satisfaction, and bully victimization. Psychology in the Schools. 2008; 45(5):446-60.

[20] Ryan RM, Frederick C. On energy, personality, and health: subjective vitality as a dynamic reflection of well-being. Journal of Personality. 1997; 65(3):529-65.

[21] Kennedy M. Personal counseling theory: hope therapy. Southern Baptist: Liberty University; 2010

[22] Alaeddini Z. [The effect of group hope therapy on the hope and mental health (Persian)]. Research in Psychological Health. 2007; 4(2):67-76.

[23] Lopez SJ, Kerr BA. An open source approach to creating positive psychological practice: a comment on Wong's strengths-centered therapy. Psychotherapy. 2006; 43(2):147-50.

[24] Keeler E, Guralnik JM, Tian H, Wallace RB, Reuben DB. The impact of functional status on life expectancy in older persons. Journals of Gerontology Series A: Biological Sciences and Medical Sciences. 2010; 65(7):727-33.

[25] Irving LM, Snyder C, Crowson Jr JJ. Hope and coping with cancer by college women. Journal of Personality. 1998; 66(2):195-214

[26] Snyder C, Cheavens J, Sympson SC. Hope: an individual motive for social commerce. Group Dynamics: Theory, Research, and Practice. 1997; 1(2):107-18.

[27] Chimich WT, Nekolaichuk CL. Exploring the links between depression, integrity, and hope in the elderly. Canadian Journal of Psychiatry. 2004; 49(7):428-33

[28] Denizli S. The role of hope and study skills in predicting test anxiety levels of university students [PhD thesis]. Ankara: Middle East Technical University; 2004.

[29] Snyder C, Irving LM, Anderson JR. Hope and health. In: Snyder C, Donelson R, editors. Handbook of Social and Clinical Psychology: The Health Perspective; 1991, p. 285-305.

[30] Karami A, Shahrestani M, Doostkam M, Alimohammadi A [Effectiveness of hope-therapy on depression improvement and life quality aspects among mothers of mentally retarded children (Persian)]. Psychology of Exceptional Individuals. 2013; 9(3):93105.

[31] Ghezelsoflu M, Esbati M. [Effectiveness of hope-based grouptherapy on life quality improvement among people with positive HIV (Persian)]. Thought and Behavior in Clinical Psychology. 2012; 6(22):89-97.

[32] Farhadi A, Movahedi Y, Movahedi M. [The effectiveness of hope-based group psychotherapy on the promotion of health related quality of life in patients with cancer (Persian)]. Yafteh. 2014; 16(1):32-42.

[33] Namdari K, Molavi H, Malekpour M, Kalantari M. [The effect of cognitive hope enhancing training on character strengths of disthymic clients (Persian)]. Journal of Clinical Psychology. 2009; 1(3):21-34.

[34] Hosseinian S, Ghasemzadeh S, Niknam M. Prediction of quality of life in female teachers on the basis of emotional \& spiritual intelligence variables. Journal of Career \& Organizational Counseling. 2012; 3(9):42-60. 
[35] Best JW, Kahn J. Research methods in education. Boston: Allyn and Bacon Publication; 1993.

[36] Kahneman D, Kahneman D, Tversky A. Experienced utility and objective happiness: a moment-based approach. In: Kahneman D, Tversky A, editors. Choices, Values and Frames. New York: Cambridge University Press; 2000, p. 673-92.

[37] Myers DC. Close relationships and quality of life. In: Kahneman D, Diener E, Schwarz N, editors. Well-Being: Foundations of Hedonic Psychology: Foundations of Hedonic Psychology. New York: Russell Sage Foundation; 1999, p. 376-93.

[38] Diener E, Fujita F. Resources, personal strivings, and subjective well-being: a nomothetic and idiographic approach. Journal of Personality and Social Psychology. 1995; 68(5):926-33.

[39] Malakouti K, Fathollahi P, Mirabzadeh A, Salavati M, Kahani S. [Validation of Geriatric Depression Scale (GDS-15) in Iran (Persian)]. Research in Medical Sciences. 2006; 30(4):361-69.

[40] Snyder C. Hope and depression: a light in the darkness. Journal of Social and Clinical Psychology. 2004; 23(3):347-51.

[41] Snyder CR. Handbook of hope: theory, measures, and applications. New York: Academic Press; 2000

[42] Spar JE, La Rue A. Concise guide to geriatric psychiatry. Virginia: American Psychiatric Association Publication; 1990.

[43] King CR, Hinds PS. Quality of life: from nursing and patient perspectives. Burlington, Massachusetts: Jones \& Bartlett Publishers; 2011.

[44] Strine TW, Chapman DP, Balluz LS, Moriarty DG, Mokdad $\mathrm{AH}$. The associations between life satisfaction and health-related quality of life, chronic illness, and health behaviors among US community-dwelling adults. Journal of Community Health. 2008; 33(1):40-50.

[45] Cieslak EN. Hope in psychotherapy process and outcome [PhD thesis]. Erie: Gannon University; 2008.

[46] Sadock BJ, Sadock VA. Kaplan and Sadock's synopsis of psychiatry: behavioral sciences/clinical psychiatry. Philadelphia: Lippincott Williams \& Wilkins; 2011. 
